\title{
The Study of the Voltage Quality Improvement Based on the Genetic PI and Repetitive Control by Using DVR
}

\author{
Penglong Yang, Wenlei $\mathrm{Li}^{*}$, Jia Zhao, Yan Ding and Jiayi Yuan \\ College of Information Science and Engineering \\ Ningbo University, 315211 China \\ E-mail:liwenlei@nbu.edu.cn
}

\begin{abstract}
In order to improve the typical voltage quality problems in distribution power system with the dynamic voltage restorer (DVR), some works are conduced in this paper. Firstly, according to the DC side voltage fluctuation of the inverter in DVR, the DC voltage is treated as a state variable and detected in real-time by the feed-forward loop. So the gain of inverter bridge is compensated for and the influence of the fluctuation of the DC input voltage on the compensatory output of the DVR system is offset. Secondly, for the deficiency of the traditional proportional integral (PI) control strategy in the DVR, a genetic PI algorithm combining with repetitive control algorithm is proposed. Theoretical analysis testifies that this compound control algorithm can effectively improve the dynamic and static performance of the system output. Lastly, the proposed control algorithm is comparatively validated through the extensive simulation studies, and the results show that the proposed compound control method can improve the response speed of the DVR system and compensation accuracy, greatly reduce the harmonic contents of the load voltage, and effectively improve the voltage quality of the sensitive load.
\end{abstract}

Keywords: dynamic voltage restorer(DVR), power quality, compound control, genetic PI control, repetitive control

\section{Introduction}

In recent years, a large number of nonlinear loads for high efficiency control are used, which seriously affect the quality of power energy, increase the harmonic content, and cause the distortion of voltage and current [1-2]. Nowadays, the power quality problem has been widely concerned, and especially it is of great significance to improve the power quality of the sensitive load in the distribution system. In the custom power devices [1], some new equipment, such as dynamic voltage restorer (DVR), distribution static compensator (DSTATCOM) and unified power quality conditioner (UPQC) are used to solve the power quality problems of the electrical distribution system. Among them, DSTATCOM is mainly used to improve the current quality of the power supply, DVR is usually used to improve the terminal voltage quality of the sensitive loads, and UPQC is mainly used to improve the quality of voltage and current simultaneously.

DVR is the power compensation equipment based on the power electronic devices, which is connected in series between the power source and the sensitive loads. When the power supply voltage quality problems occur, such as the voltage sag, swell, flicker and harmonic distortion, by DVR injecting the compensation component into the power line, the voltage quality of sensitive loads can be effectively improved and their normal operation can be ensured also.

Voltage sag is the most common voltage quality problem, which is defined that the RMS (root mean square) voltage value suddenly drops in 0.9 to 0.1 and the duration time is in $10 \mathrm{~ms}$ to 1 minutes. Another less common distortion is the voltage swell, defined as the voltage RMS value suddenly rising to between 1.1 and 1.8 , and the duration time is

*Corresponding Author 
also in $10 \mathrm{~ms}$ to 1 minute. In addition, the harmonic content of the power supply voltage is also an important indicator of the voltage quality. As one important custom power supply equipment, DVR can effectively improve these voltage quality problems through the design of reasonable topology and control algorithm [3]. At present, the single-phase Hbridge structure is relatively independent and the control is also simple [4], so it is more used in the DVR inverter unit. After selecting the appropriate topology, the reasonable choice of control strategy is another important condition to ensure the compensation effectiveness of DVR [9]. The existed control strategies consist of some linear control methods, such as the proportional integral differential (PID), the feedback and feedforward control, and the pole assignment, etc. [4-6]. Some nonlinear control methods are also used, such as the repetitive control [4], no bad beat control [7], neural network control [8], fuzzy control [9], space vector control [10], H-infinity control [11].

In this paper, firstly, for the no series transformer type DVR which is applied in singlephase low-voltage system, the mathematical model of it is established. Then a composite control method (a genetic PI control combining with the repetitive control) is proposed. Compared with the repeated algorithm used in [4], by the increased link $K_{r} z^{K} S(z)$, the system correction control and resonance elimination can be completed at the same time, and the high frequency gain can be made to decay quickly. A large number of simulation results show that, for the main power quality problems of the power supply voltage, the proposed DVR composite controller can give fast voltage compensation, and can significantly improve the voltage quality.

The rest of the paper is organized as follows. Section 2 is devoted to the model of DVR. The design and the analysis of the compound controller are described in Section 3. The simulation studies are given in Section 4 , and followed by Section 5 which concludes the work.

\section{The Model of DVR}

In this paper, the voltage transformation system is a single phase full bridge structure, and the output compensation voltage is coupled to the main circuit through the filter capacitor $C_{f}$. Single-phase bridge DVR structure without series transformer type is shown as in Figure1.

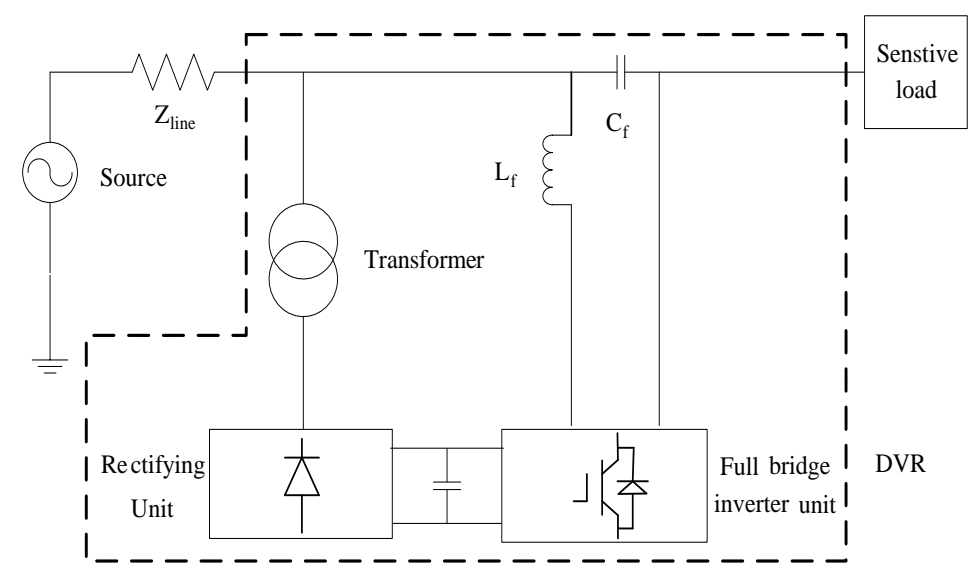

Figure 1. The Structure of DVR without Series Transformer

When the DVR is in the state of compensation, its single phase equivalent structure is shown in Figure 2. 


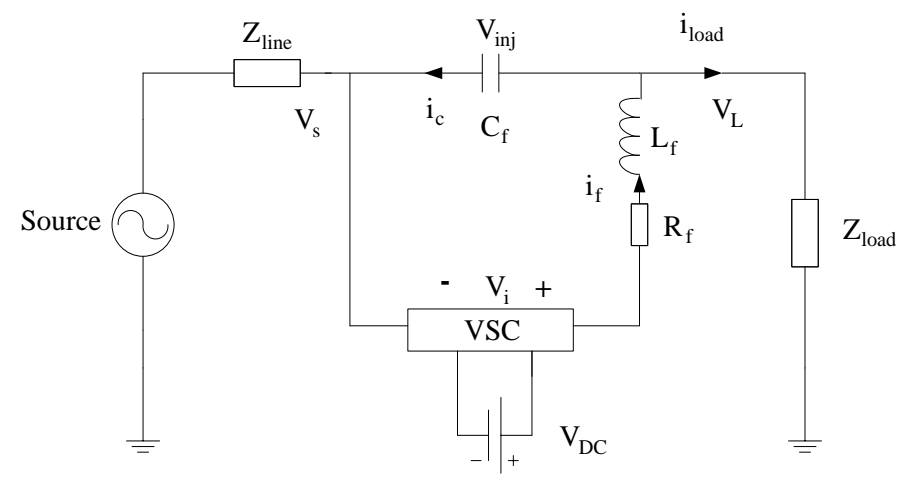
Figure 2. The Equivalent Circuit Diagram of Single-phase DVR without
Series Transformer

In Figure 2., $\mathrm{Z}_{\text {line }}$ is the equivalent impedance of power lines, $V_{s}$ is the voltage of source access point, $V_{i n j}$ is the compensation output of the DVR, $i_{c}$ is the current of the filter capacitor, $i_{f}$ is the current of the filter inductor, $i_{\text {load }}$ is the load current, $C_{f}$ is the filter capacitor, $L_{f}$ is the filter inductor, $R_{f}$ is the line resistance of filter inductance loop, $V_{i}$ is the compensation voltage output by the voltage source converter (VSC) in DVR, $V_{D C}$ is the DC side voltage of the VSC. Then the state equations of the single-phase DVR are in the following.

$V_{L}=V_{S}+V_{i n j}$

$i_{f}=i_{c}+i_{\text {load }}$

$i_{c}=C_{f} \frac{d V_{i n j}}{d t}$

$V_{i}=R_{f} i_{f}+L_{f} \frac{d i_{f}}{d t}+V_{i n j}$

The fluctuations of the DC side voltage $V_{D C}$ in the actual DVR system can be simulated by a fluctuating voltage source. The gain coefficient of VSC is $K_{m}$. The block diagram of the open-loop control system can be get from the state equations of the single phase DVR, which is shown in Figure 3.

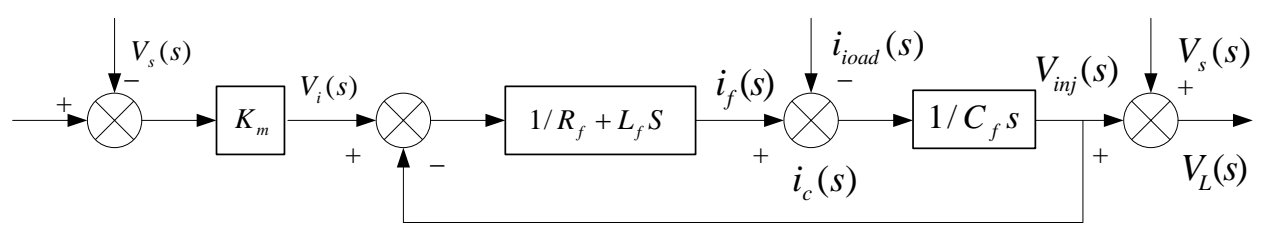

Figure 3. The Open-loop Control System of Single Phase DVR

The analysis shows that the open loop transfer function of the DVR main circuit is as follows.

$G(s)=\frac{k_{m}}{L_{f} C_{f} s^{2}+R_{f} C_{f} s+1}$ 


\section{The Compound Control Method of DVR}

\subsection{Genetic PI Control}

By the open loop transfer function of the DVR main circuit and according to the actual value of the system, the structure of the traditional PI control algorithm is shown in Figure 4. The PI controller parameters $K_{p}$ and $K_{i}$ can be determined by using the $Z-N$ critical method. In this paper, $K_{p}=1$, and $K_{i}=100$. However, because the transfer function has certain deviation with the actual system, and DVR is a nonlinear device, so the control effect is not ideal.

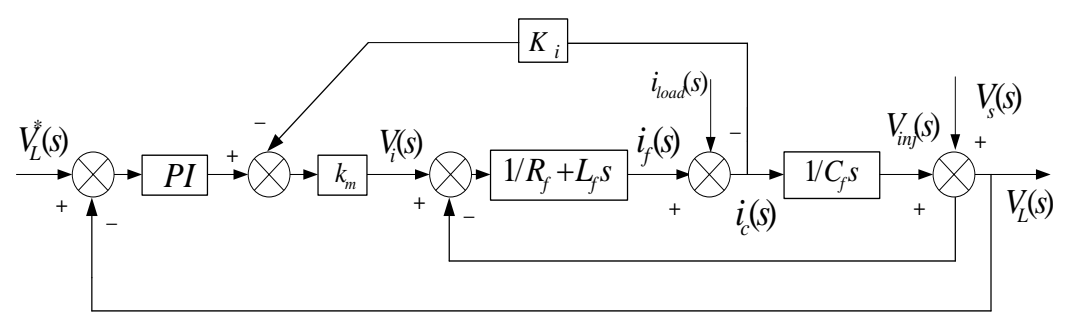

\section{Figure 4. The Double Closed-loop Control Structure}

Genetic algorithm (GA) is an automatic optimization method [12], which is based on the principle of the natural selection and biological inheritance. By simulating the evolution and variation ability of the nature biological population, GA searches for the set goals by the adaptive probabilistic. GA is also a kind of method to directly manipulate the structure object. It owns good characteristics of target optimization, because it can adjust the search direction adaptively and does not need to be determined by the rules. Even if the model of the controlled object can not be determined, the system control parameters can be optimized based on the output of the object, and the control objectives can be met. In this paper, based on the traditional double closed loop PI control, the GA is applied in the PI control, and the control parameters are optimized to obtain a better system performance. The realization process of genetic PI algorithm is as follows.

Firstly, the objective function including parameters $K_{p}$ and $K_{i}$ (Fitness function) should be founded, and the fitness value of every combination in searching domain should be computed.

Secondly, the selection, crossover and mutation operation of the combination in groups are carry out until the optimal adaptation degree in searching group is found and the optimal target value is get. The system control configuration of the genetic PI controller is shown in Figure 5.

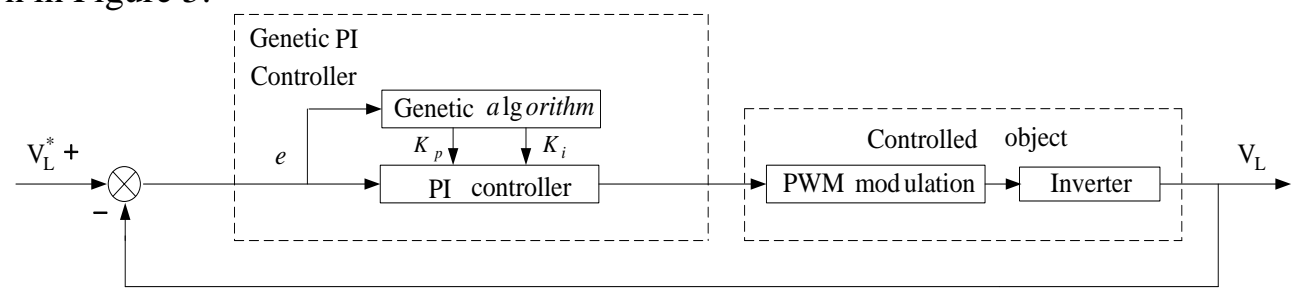

Figure 5. The Structure of PI Control System using GA

In this paper, we choose the integral of time multiplied absolute error (ITAE) performance index as the objective function:

$J=\int_{0}^{\infty} t|e(t)| d t$ 
The goal of genetic optimization is to find the minimal value of the fitness function. Therefore, it is necessary to rewrite the objective function, i.e., the minimum value to be calculated will be changed into the maximum, and then the object function can be taken as the reciprocal of ITAE. So the fitness function is as follows:

$$
f(t)=\frac{1}{I T A E}=\frac{1}{\int_{0}^{\infty} t|e(t)| d t}
$$

\subsection{Repetitive Control}

The PI control strategy based on the genetic algorithm can get better control parameters, and further improve the dynamic performance of the DVR system and the precision of the voltage compensation. But there are some limitations when PI control is applied in the nonlinear object DVR, especially the PI algorithm can not give a good performance when the voltage quality is poor in the controlled DVR system. Therefore, on the basis of the existed algorithm, the improved algorithm is needed to enhance its compensation performance. So the repetitive control algorithm are added in this paper because of its advantage of control precision, and the repetitive control method based on internal model principle can eliminate periodic error in the stable closed loop control. When the repetitive control is used in DVR systems, the difference of given signal and feedback signal is as a tracking signal in each switching cycle, which was added to the original control signal, then the repetitive distortion in the output signal of the DVR system can be eliminated [21].

From the Fourier analysis, it can be known that the harmonic signals in the power supply voltage are formed by the superposition of a variety of different frequencies and amplitude of the AC signals. According to the repetitive control principle, it is needed to have an internal model in the design model, which can reflect the dynamic characteristics of the harmonic signals in system input voltage, and then the suppression of each harmonic in the voltage can be realized. But it is very complicated to design a dynamic model for each kind of frequency AC signal in harmonics, and it is not desirable. Taking into account the harmonic signal, the different frequency AC signal in each fundamental cycle will be exactly repeated as the same waveform. Therefore, the fundamental period can be used as the repetition period of the system input signal, and the dynamic internal model of the DVR is designed as follows.

$G_{m}(s)=\frac{1}{1-e^{-L s}}$

where $L$ is the $20 \mathrm{~ms}$ fundamental cycle. The discrete form of (8) in the digital control is in the following.

$G_{m}(z)=\frac{1}{1-z^{-N}}$

where $N$ is the sample number per cycle.

Due to the limitation on the principle of repetitive controller, there is a fixed fundamental wave cycle delay in the control signal. In order to guarantee the response speed of the compensation voltage in DVR system, the embedded structure is adopted here. That is, the feed-forward reference signal in input voltage is superimposed on the original control in the DVR control instruction of the closed-loop system. The block diagram of repetitive control system is shown in Figure 6. 


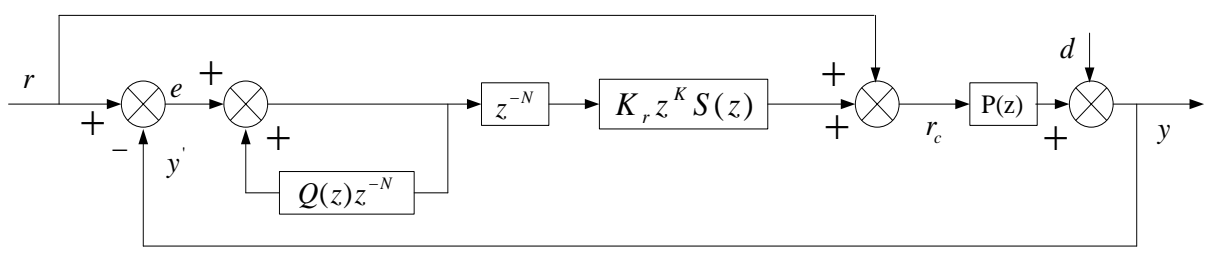

Figure 6. The System Block Diagram with Repetitive Control

In Figure $6, r$ is the reference signal, $e$ is the error signal, $r_{c}$ is the compensated reference signal, $d$ is the external disturbance signal, $y$ is the output value of the control system. $Z^{N}$ is the delay link, $N$ is the sampling frequency in each fundamental period, $Q(z)$ is the factor of low pass filter, $K_{r}$ is the repetitive control gain, $z^{K}$ is the phase compensation link, $S(z)$ is the filter, $P(z)$ is the controlled object transfer function. We have:

$$
\frac{E(z)}{D(z)}=\frac{1}{1+\frac{z^{-N}}{1-Q(z) z^{-N}} S(z) P(z)}=\frac{1-Q(z) z^{-N}}{1-z^{-N}(Q(z)-S(z) P(z))}
$$

Supposed $Q(z)=1$ and $P(z)$ is stable, the stability condition of the closed-loop system is

$$
\|1-S(z) P(z)\|<1, z=e^{j w T}, 0<\omega<\pi / T
$$

where $T$ is the sampling time.

For the closed-loop system in Figure 6, if the angular frequency $\omega_{m}$ of the disturbance $d$ and reference input signal frequency have the following relations,

$\omega_{m}=2 \pi n f, m=0,1,2 \ldots . M$

where $M=N / 2$ when $N$ is a even, and $M=(N-1) / 2$ when $N$ is a odd, then we have

$z^{-N}=1,\|E(z) / D(z)\|=0$

This shows that the repetitive controller can eliminate any harmonic in theory, and the system can achieve the error free tracking after adding the repetitive control. The assumption of $Q(z)=1$ on the stability requirements of $P(z)$ is relatively high. For the actual system this condition can be weaken, and $Q(z)$ can be set to low pass filter or a constant.

The following equation can be derived from the stability analysis of Figure 6 .

$$
\begin{aligned}
& E(z)=R(z)-Y(z)+D(z) \\
& Y(z)=P(z)\left[R(z)+E(z) \frac{z^{-N}}{1-Q(z) z^{-N}} S(z)\right]
\end{aligned}
$$

From (14) and (15), we have:

$$
E(z)=E(z) z^{-N}[Q(z)-P(z) S(z)]+[R(z)-P(z) R(z)+D(z)]\left[1-Q(z) z^{-N}\right]
$$

If for all $\omega$, the following equation is satisfied,

$$
|Q(z)-P(z) S(z)|_{Z=e^{j m}}<1,
$$

and $P(z)$ is stable, then $e(k)$ is bounded, and the system is stable at this time. In order to overcome the influence of the object model, the $Q(z)$ is generally selected as a function whose module is less than 1 and close to 1 , or a constant of less than 1 . In this paper, the 
sampling frequency is $20 \mathrm{kHz}$, the fundamental frequency of the power grid is $50 \mathrm{~Hz}$, so $\mathrm{N}=20000 / 50=400$.

In order to simplify the design, we take $Q(z)=0.98$. The design of repetitive controller is actually the design of $\mathrm{Krz}^{K} S(z)$. $S(z)$ is mainly used to eliminate the resonance peak, and make the high frequency gain be attenuated fast. $S(z)$ is composed of a notch filter $S_{1}(z)$, which is used to eliminate the resonance peak of $P(z)$, and the two order low-pass filter $S_{2}(z)$ is used for the high frequency attenuation. Notch filter is also known as the zero phase shift filter, and it can achieve strong attenuation of the specific frequency, but has small influence to the value around notch frequency. The transfer function is as follows.

$S_{1}(z)=\frac{z^{m}+a+z^{-m}}{2+a}$

(

Substitute $Z=e^{j \theta}$ into (18), we have

$S_{1}(\theta)=\frac{2 \cos m \theta}{2+a}$

where $S_{1}(\theta)$ is a real number, when $a \in(0,2), S_{1}(\theta)$ is negative or positive, so the phase angle of $S_{1}(z)$ is changed between $0 \sim \pi$. When $a>2$ or $a=2$, the phase change of $S_{1}(z)$ is zero. It is shown that $S_{1}(z)$ has a strong attenuation for the specific frequency.

We also have

$2 \cos m \theta+2=0$

If

$\omega=20000 \pi / m$

By substituting $\omega=1 / \sqrt{L C}$ into (21), we get $m=11.92$. If $m=12$, then (18) is transformed into:

$S_{1}(z)=\frac{z^{12}+2+z^{-12}}{4}$

The function of $S_{2}(z)$ is used to suppress high order harmonics, which is essentially a low pass filter, and the cutoff frequency is a value between the resonant frequency and the cutoff frequency of the $L C$ filter. In this paper, the $S_{2}(z)$ is taken as:

$S_{2}(z)=\frac{0.02008+0.04016 z^{-1}+0.02008 z^{-2}}{1-1.56102 z^{-1}+0.64135 z^{-2}}$

The value of $K_{r}$ is used to adjust the convergence rate of the repetitive controller, which is generally positive constant of less than 1 and can be obtained. The role of $z^{K}$ is to compensate for the lag phase. We take $K_{r}=0.8$ and $K=6$ by trails and errors.

\subsection{The Compound Control}

Due to the genetic PI control takes effect for the switching frequency, and the repetitive controller works for the fundamental cycle. So the action time of the two controllers is not the same and the speed of response is not consistent. They work independently of each other in the steady state, and they are not affected by each other. When the DVR runs in a stable state, the feedback error becomes small, and the regulation effect of genetic PI becomes weak, then the repetitive controller provides the main regulation function at this time. When the large disturbances occur, the error will suddenly become large, the genetic 
PI controller will take effect immediately and regulate the steady-state error of voltage after receiving the real-time error changes, but the repetitive controller will not immediately regulate the DVR because of a fundamental cycle delay. So it is possible to adopt a composite control method to combine the advantages of the two methods. The dynamic characteristics of DVR system can be improved by the genetic PI control, and the compensation precision of DVR output can be improved by the repetitive control

The block diagram of the compound control is shown in Figure 7. The error signal $e$ enters the genetic PI control and repetitive control at the same time, then the instruction signal is generated under the combined action of the two algorithms. The genetic PI control is mainly to improve the dynamic response characteristics of the DVR system, and the repetitive controller is mainly to reduce the distortion caused by periodic disturbance in the system and the harmonic contents of the compensation voltage. Besides, the feedforward control is used for the fluctuations of DC side voltage $V_{D C}$ in the VSC. After the correcting of the command signal output by the genetic PI and the repetitive algorithm, the gain of the inverter can be compensated for, and the negative effect on the compensation voltage of the DVR output is reduced. Finally, the compensation of DVR output is controlled by the control signal.

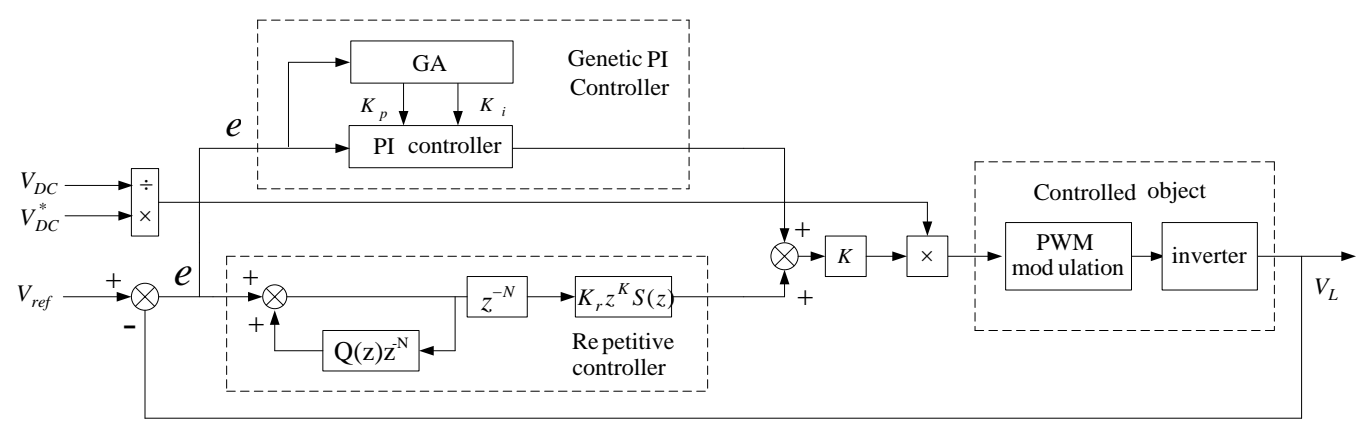

Figure 7. The Block Diagram of Compound Control

\section{Simulation Study}

The simulation model of DVR system is shown in Figure 8. The A phase is taken as the DVR single-phase voltage source from the programmable three-phase power supply, then the main voltage quality problems, such as voltage sag, swell and harmonics can be simulated. DVR is connected between the power supply and the sensitive load. The output voltage of the voltage source converter is coupled to the distribution line by the capacitance from the $L C$ filtering, and provides a normal voltage to the loads.

In the simulation study, for the cases of the power voltage sag, swell, and whether it contains harmonics or not, and the case of the sensitive load adaptability for the voltage quality problems, the proposed hybrid control algorithm is compared with the traditional PI control. Need to explain is that the simulation of the $30 \%, 60 \%, 90 \%$ of the voltage changes have been studied, but because the compensation effect is basically consistent and the limit of the paper length, we only give the results of $60 \%$. In addition, all the variables in the DVR simulation system are normalized, and the pu value is adopted. 


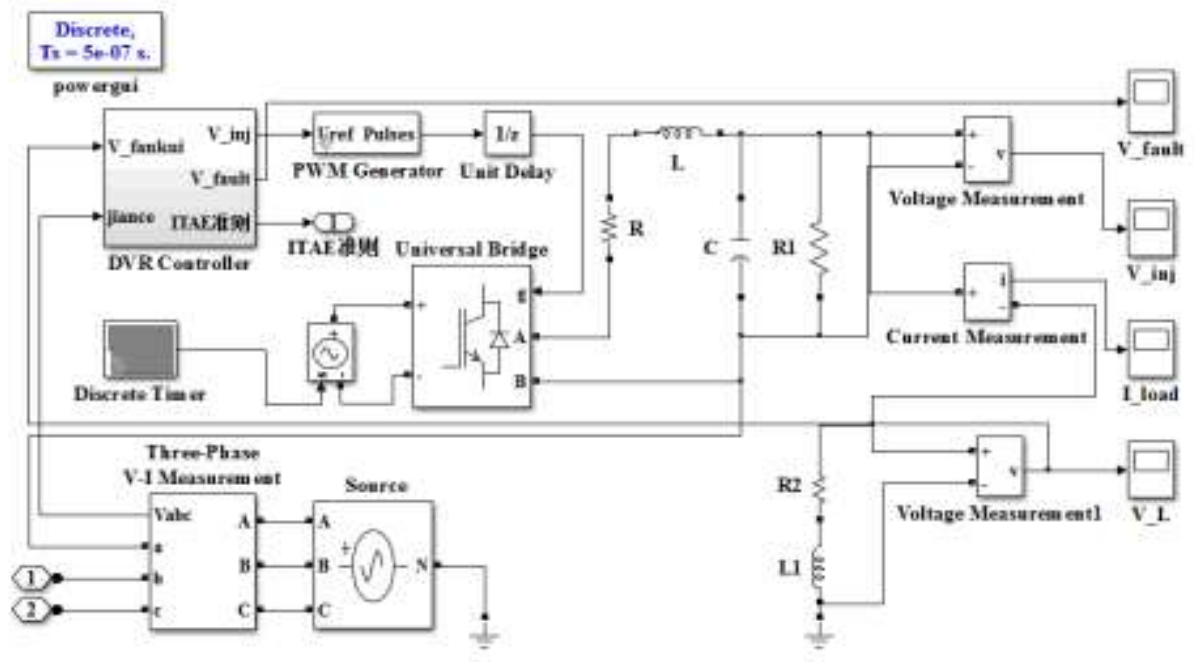

Figure 8. The Simulation Model of the DVR System

\subsection{Compensation Analysis of the Voltage without Harmonics}

When the voltage variation is $60 \%$, that is, the $60 \%$ depression and the $60 \%$ voltage swell occurred in $0.05 s$ and $0.18 s$, and sustained four fundamental cycles as well, respectively. The DVR system is studied under two cases: the traditional PI control when the DC side fluctuation is without control and the compound control when the DC side fluctuation with feed-forward control.

The DC side fluctuation of the analog converter is shown Figure 9, and the fluctuation change of the voltage is $20 \%$.

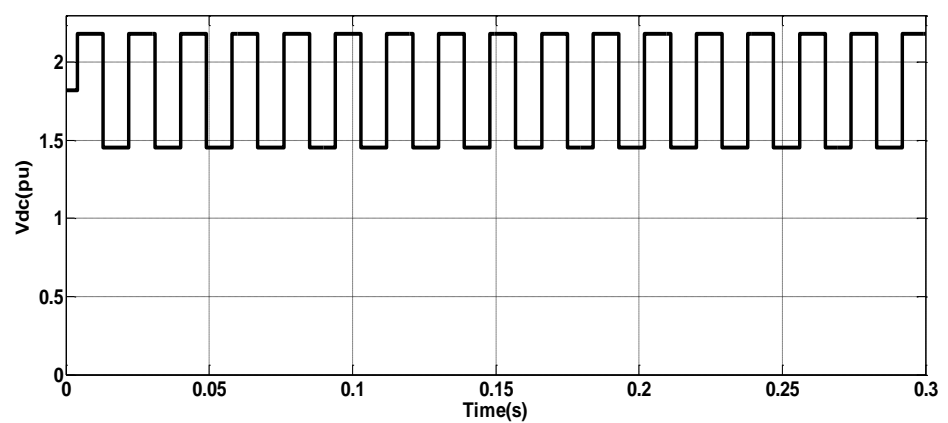

Figure 9. The Voltage Fluctuation of the DC Side of Inverter

The waveform of the power source voltage with $60 \%$ changes is given in Figure 10(a) and the contractive waveforms of the compensation output under the two different control methods are shown in Figure 10(b). Obviously, the compensation waveform of the hybrid control strategy proposed in this paper is better than that of the traditional PI control, because the compensation precision is higher, and the compensation speed of the output voltage is also slightly higher.

Figure 10 also gives the waveform of the sensitivity load voltage, the error voltage, and the harmonic analysis in the sensitive load voltage under the two different control algorithms in DVR compensation system. From Figure 10(c) we can see the load terminal voltage during the power supply depression under the traditional PI control did not reach to the rated value, and there are some errors and an obvious distortion. From 10(d), the rated load voltage value is compensated by the compound control and the waveform is completed. 


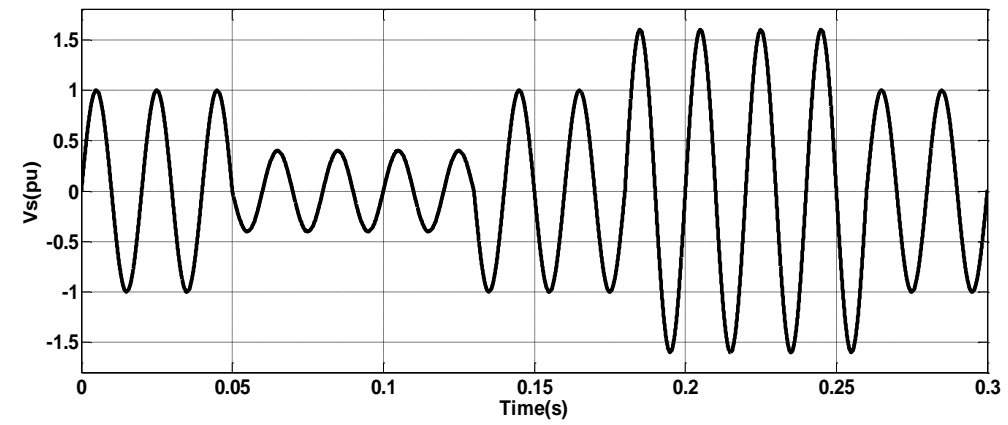

(a) The waveform of supply voltage changing $60 \%$

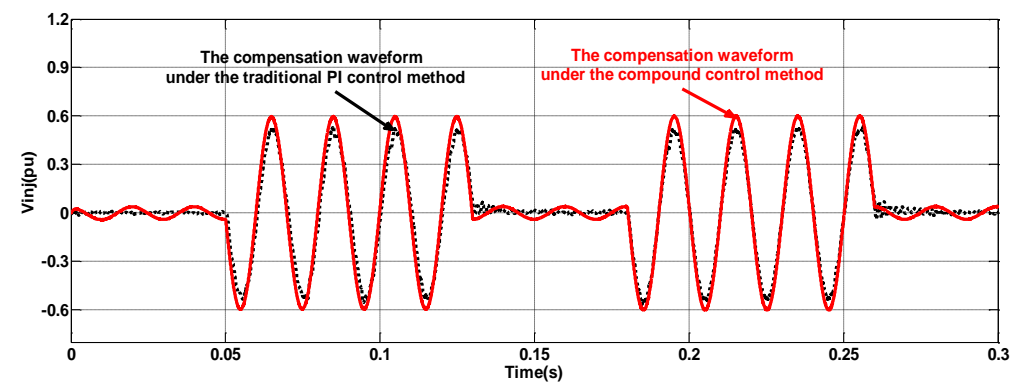

(b) The contrastive compensation waveform under the two different control methods

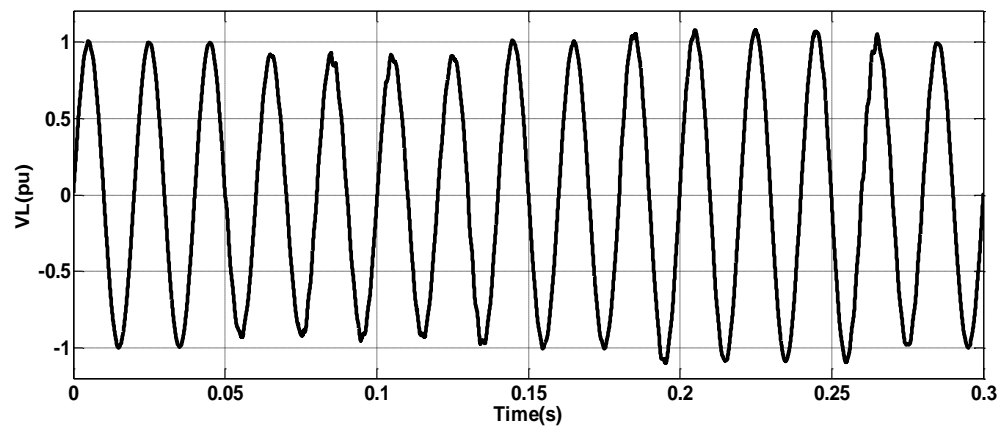

(c) The load voltage with the traditional PI control method

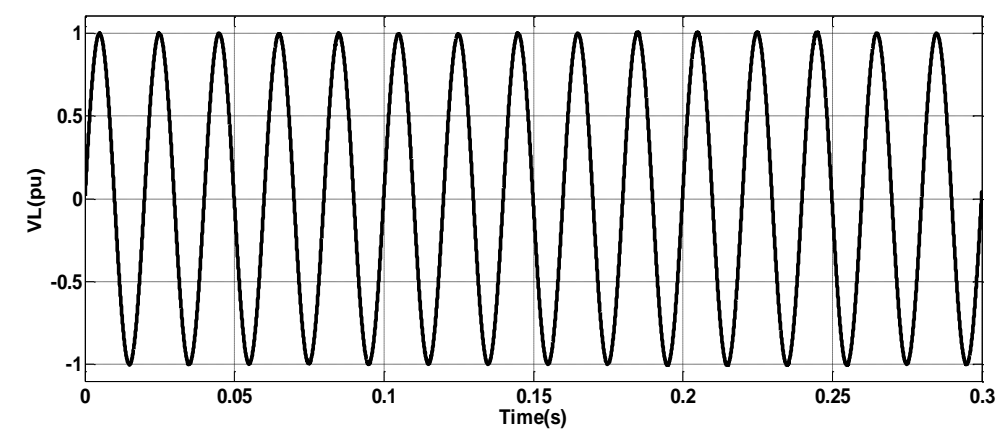

(d) The load voltage with the compound control method 


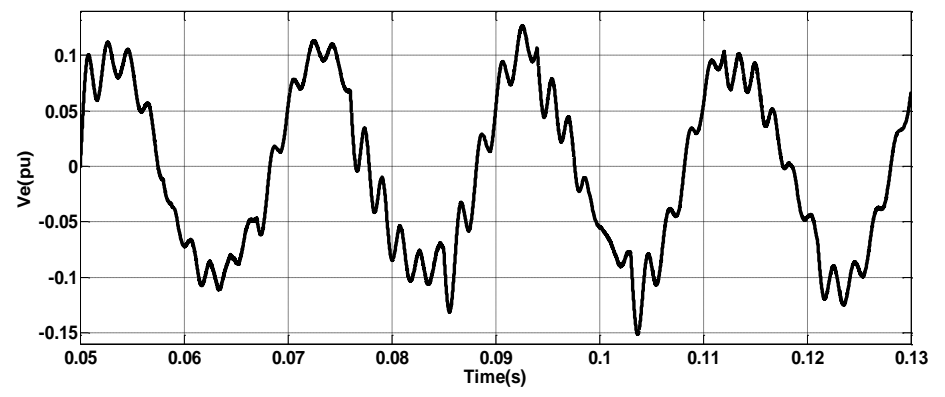

(e) The error of load voltage with the traditional PI control method

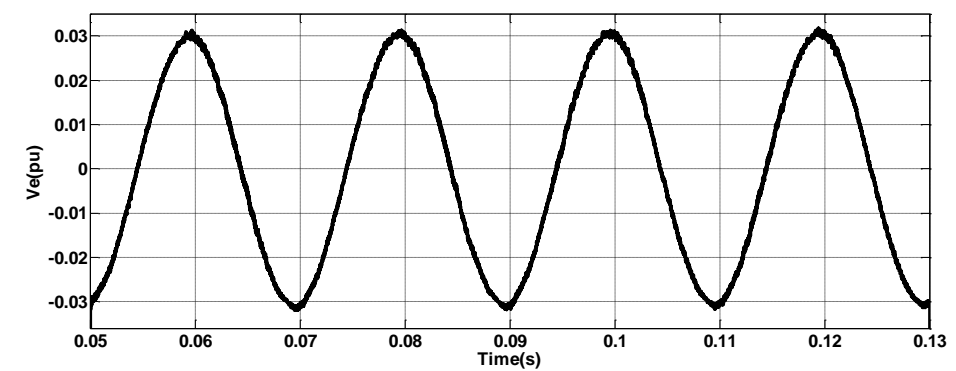

(f) The error of load voltage compensated with the compound control method

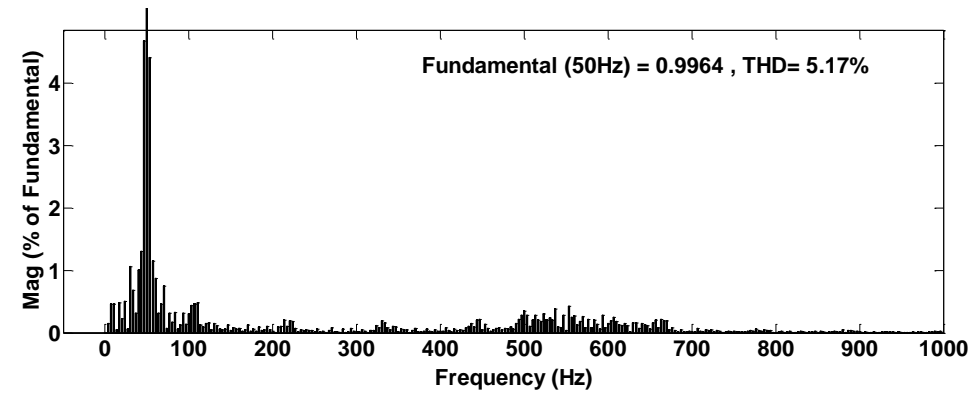

(g) The harmonic analysis of load voltage with the traditional PI control method

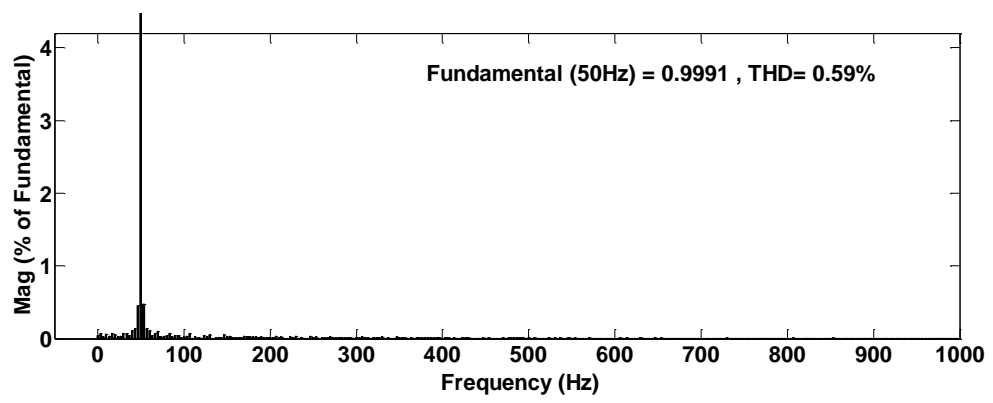

(h) The harmonic analysis of load voltage with the compound control method

\section{Figure 10. Simulation Contrastive Waveforms of $60 \%$ Variation in the Supply Source}

Besides, by the comparison waveforms of Figure 10(e), 10(f) and Table 1, it can be seen the fluctuations of load terminal voltage error peak is around 0.13 under the traditional PI control, but it decreased obviously under the control of composite control, and the fluctuations is in about 0.031. From Figure 10(g), 10(h) and Table 1, the load 
voltage waveform distortion under the traditional PI control is larger, and the total harmonic distortion rate (THD) is 5.17\%, while THD rate under the composite control is decreased to $0.59 \%$. So the hybrid control strategy proposed in this paper has the higher precision of voltage compensation.

Table 1. Compensation Comparison of the Voltage without Harmonics

\begin{tabular}{|c|c|c|}
\hline Control method & The error peak of load voltage & Total harmonic distortion \\
\hline Traditional PI control & around 0.13 & $5.17 \%$ \\
\hline Compound control & around 0.031 & $0.59 \%$ \\
\hline
\end{tabular}

\subsection{Compensation Analysis of the Voltage Sag, Swell and with Harmonics}

When the variation of power supply voltage is $60 \%$, that is, the $60 \%$ depression of power supply voltage occurred in $0.05 \mathrm{~s}$ and sustained four fundamental cycles. The $60 \%$ swell occurred in the $0.18 \mathrm{~s}$ and continued four fundamental cycles. At the same time, the $5^{\text {th }}$ to $8^{\text {th }}$ harmonics are injected into the power supply voltage. In the case that the power quality is poor, it can be seen from the comparative analysis that the proposed control algorithm still can give satisfactory results shown in Figure 11. Figure 11(a) is the waveform of the supply voltage with $60 \%$ changes and harmonics, at this time, the voltage quality is indeed very poor. From Figure 11(b), it can be seen the load voltage waveform distortion is serious under the traditional PI control, and the compensation effect is poor as well. Figure 11(c) shows the load voltage waveform under the composite control is relative good, and it is significantly better than that of 11(b).

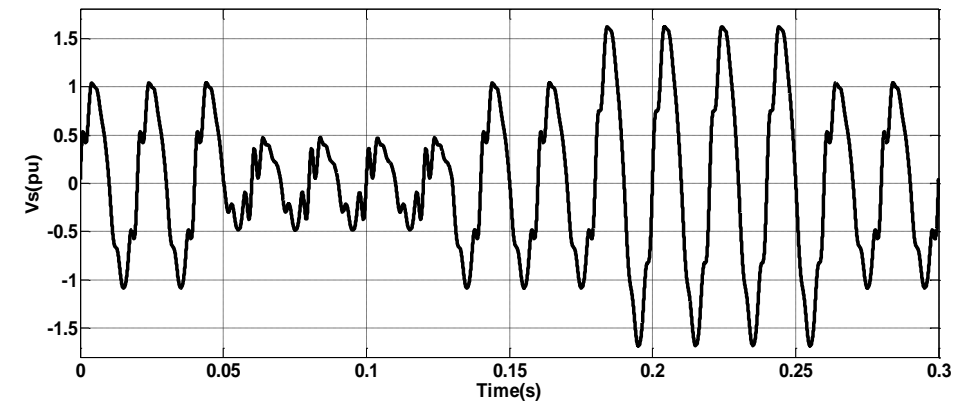

(a) The Waveform of Supply Voltage Changing 60\% Amount and Containing Harmonic Quantity

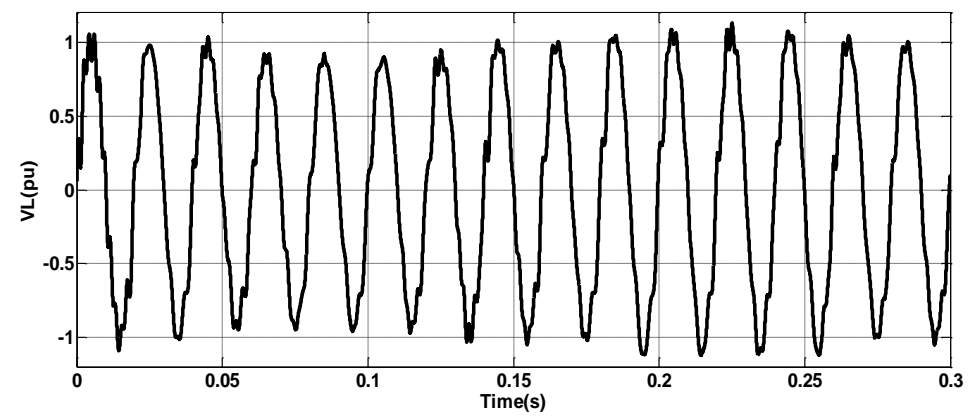

(b) The Load Voltage with the Traditional PI Control Method 


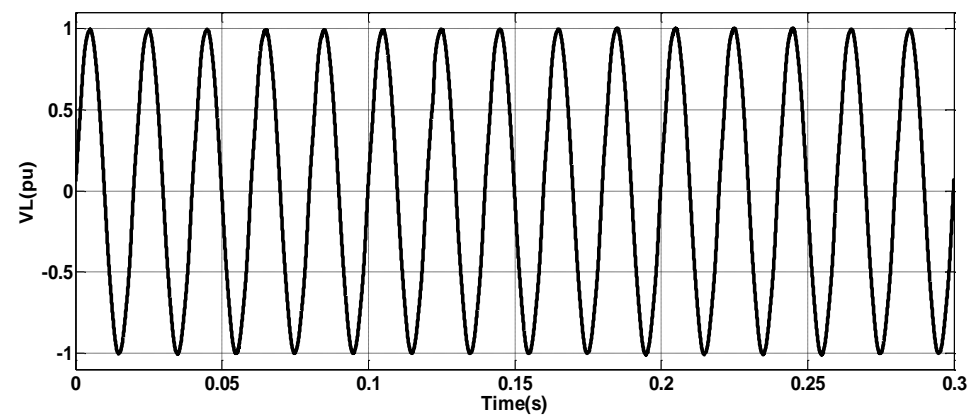

(c) The Load Voltage with the Compound Control Method

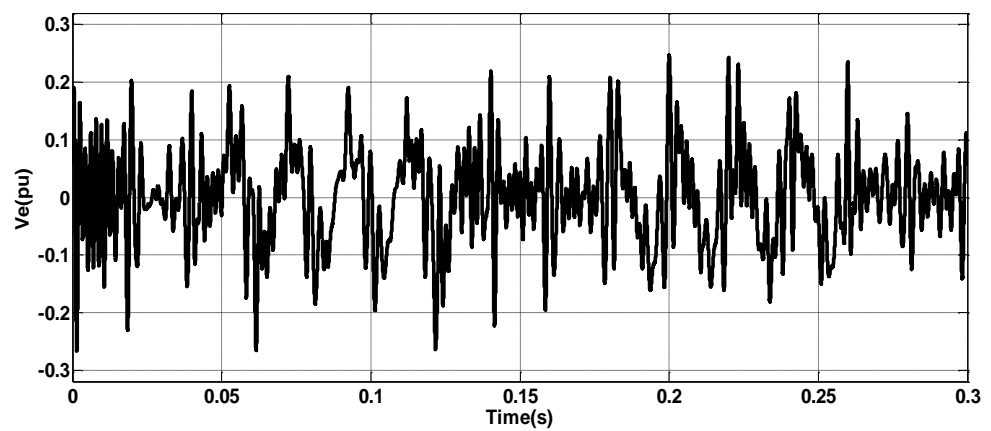

(d) The Error of Load Voltage with the Traditional PI Control Method

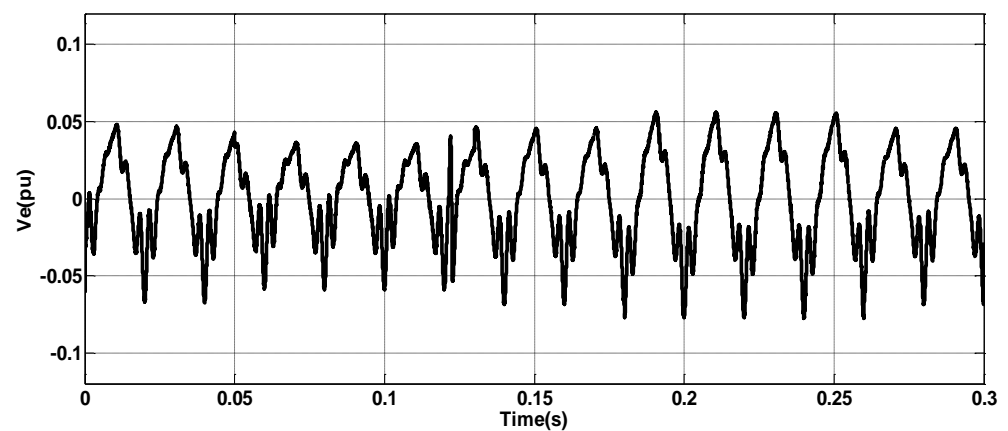

(e) The Error of Load Voltage Compensated with the Compound Control Method

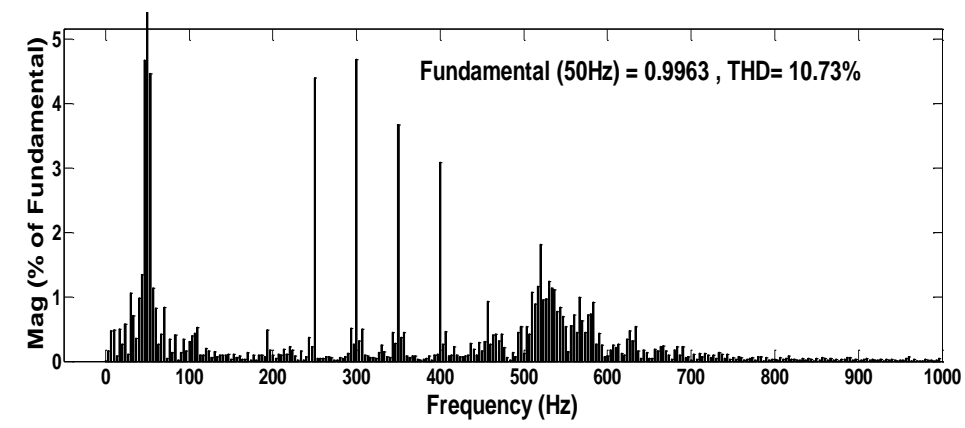

(f) The Harmonic Analysis of Load Voltage with the Traditional PI Control Method 


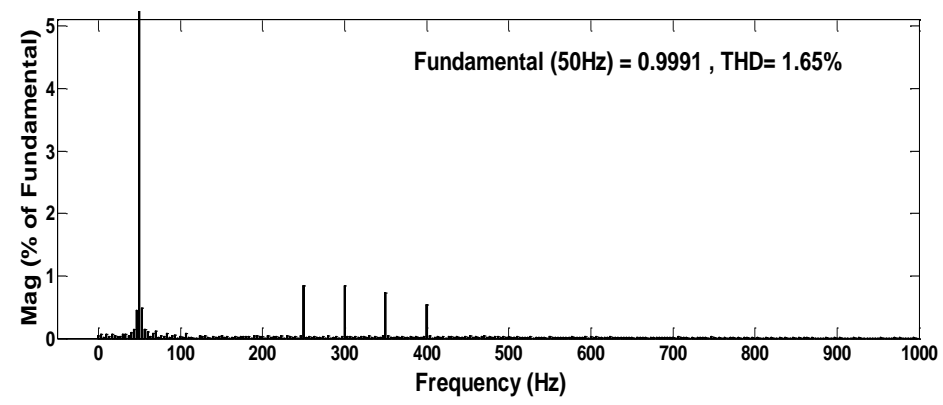

(g) The Harmonic Analysis of Load Voltage with the Compound Control Method

\section{Figure 11. Simulation Contrastive Waveforms of $60 \%$ Variation and Containing Harmonic in the Supply Source}

From the comparison results of Figure 11(d), 11(e) and Table 2, the load terminal voltage error peak under the traditional PI control fluctuates between 0.2 to 0.3 , but load terminal voltage stabilization error peak under the control of composite decreased obviously, only fluctuates between 0.05-0.06 and the control accuracy is better than that of Figure 11(d). From Figure 11(f), 11(g) and Table 2, the load side voltage waveform distortion under the traditional PI control is relatively large, the THD is of $10.73 \%$, and it can not meet the required standard voltage quality. But the THD of the compound control method is of $1.65 \%$, still meets the required standard quality. The analysis results show that the proposed hybrid control algorithm can still ensure the voltage quality of the load side even if the voltage quality is very poor.

Table 2. Compensation Comparison of the Voltage Sag, Swell and with Harmonics

\begin{tabular}{|c|c|c|}
\hline Control method & The error peak of load voltage & Total harmonic distortion \\
\hline Traditional PI control & between 0.2 to 0.3 & $10.73 \%$ \\
\hline Compound control & between 0.05 to 0.06 & $1.65 \%$ \\
\hline
\end{tabular}

\subsection{Load Adaptability Analysis}

The simulation is carried out to DVR system with the sensitive load also. The sensitive load is composed of a diode rectifier and a voltage regulator (DC/DC converter). The AC voltage is transform to the $\mathrm{DC}$ voltage by $\mathrm{AC} / \mathrm{DC}$ rectifier, and then it will be converted into a DC power supply for the electrical equipment by the DC/DC converter. If the AC power supply voltage drops, the DC side voltage of AC/DC rectifier will also be reduced. When this voltage drops too much and can not meet the requirements of power equipment, the sensitive load will not work properly.

When the supply voltage has the temporary $60 \%$ sag, that is, at $0.05 \mathrm{~s}$ the $60 \%$ depression occurs, sustains 10 fundamental cycles, and the voltage returns to the normal at $0.25 \mathrm{~s}$. The voltage of the DC side of the AC/DC converter after the voltage drop without control is shown in Figure 12. It can be known that the DC side voltage drop at this time is very large, which will cause the computer and the electronic equipment to be unable to work. 


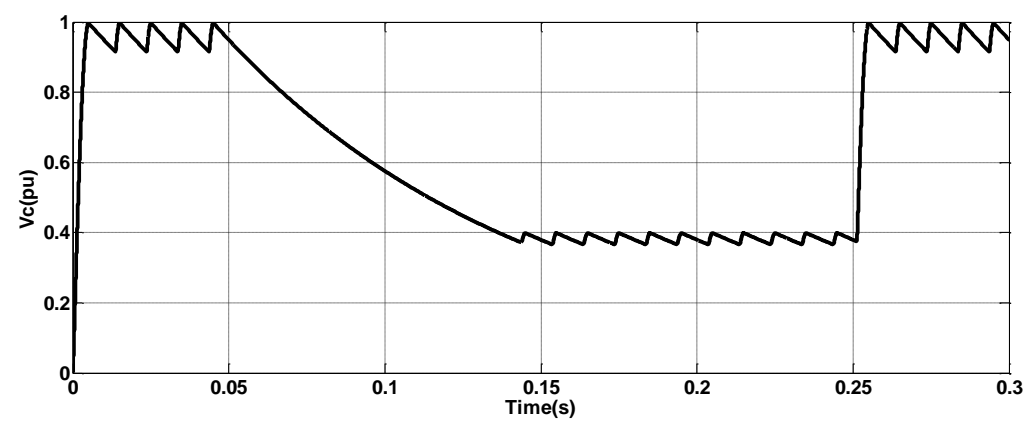

Figure 12. The Voltage Waveform of Converter dc Side with Voltage Drop

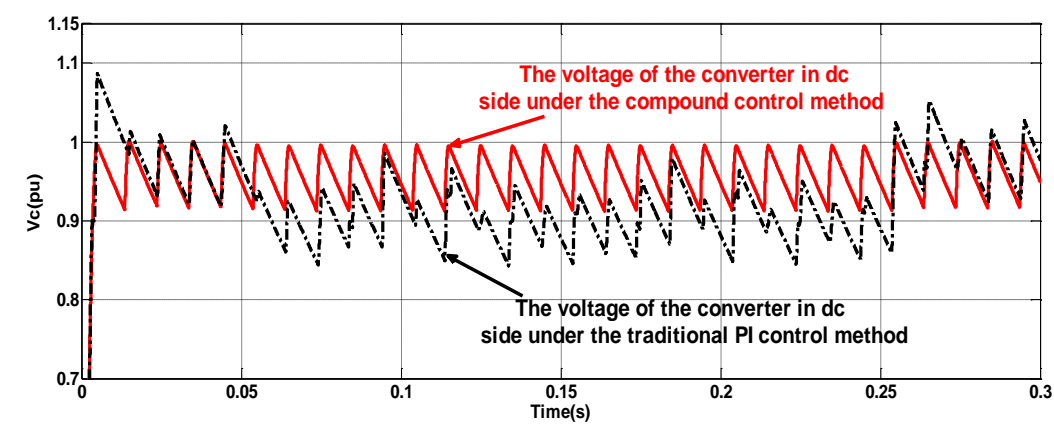

Figure 13. The Contrastive Voltage of the Converter in dc Side under the Two Control Methods

In view of the above situation, when the traditional PI strategy and the compound control method are used in the DVR system with sensitive load, the contrastive waveforms of the DC side voltage of the converter are shown in Figure 13. It can be seen that the DC side voltage of the rectifier is able to pick up under the two kinds of control methods, but the waveform under the composite control is more stable than that of the PI control, and the fluctuation range is smaller, so the voltage stability of the sensitive load can be kept under the condition that the voltage quality is poor, and the normal operation of the sensitive load is not affected.

\section{Conclusion}

In this paper, according to the model of DVR and the analysis of the double closed loop feedback control, there is the control error and poor anti-interference ability in the feedback loop with only the PI control, so the composite control strategy is proposed, which is combined with the respective advantages of the genetic PI control and the repetitive control. In the composite control, the genetic PI control is used to improve the response speed and the dynamic performance of the system, and the repetitive control is used to improve the output compensation accuracy of DVR system, and reduce the harmonic contents. The simulation analysis show the proposed control method in this paper has more advantages in the compensation speed, output compensation precision and reducing the harmonics. The sensitive load adaptability analysis is also conducted. It can be drawn that, compared with the conventional PI control, the DVR system under the composite control has better dynamic and static performance, and owns the stronger ability to suppress harmonics. When the quality problems of the power supply voltage appear, the sensitive load can still work properly. 


\section{Acknowledgments}

This work was supported in part by K. C. Wong Magna Fund in Ningbo University, the Zhejiang Open Foundation of the Most Important Subjects (XKXL1528).

\section{References}

[1] A. Domijan, A. Montenegro, A. J. Keri, et al., "Custom power devices: an interaction study" , IEEE Transactions on Power Systems, vol. 20, no. 2, (2005), pp. 1111-1118.

[2] B. Vairamohan, W. Komatsu, M. Galassi, et al., "Technology assessment for power quality mitigation devices - Micro-DVR case study”, Electric Power Systems Research, vol. 81, no.6, (2011), pp. 12151226.

[3] R. Omar, N. A. Rahim, "New Configuration of a Three Phase Dynamic Voltage Restorer (DVR) for Voltage Disturbances Mitigation in Electrical Distribution System", Arabian Journal for Science and Engineering, vol. 37, no.8, (2012), pp. 2205-2220.

[4] Z. X. Huang, X. D. Zou, T. Li, et al., "Research on current control mode single-phase dynamic voltage regulator based on pole-assignment and repetitive control", Transactions of China Electrotechnical Society, vol.27, no.6, (2012), pp. 252-260.

[5] J. Wang, A. Q. Xu, G. Q. Weng, Y. Y. Shen, et al., "A survey on control strategy of DVR", Power System Protection and Control, vol. 38, no.1, (2010), pp. 145-150.

[6] G. A. Carlos, E. C. Santos, C. B. Jacobina, et al., "Dynamic Voltage Restorer Based on Three-Phase Inverters Cascaded Through an Open-End Winding Transformer", IEEE Transactions on Power Electronics, vol.31, no.1, (2016), pp. 188-199.

[7] H, Kim, S. Sul, "A Novel Filter Design for Output LC Filters of PWM Inverters", Journal of Power Electronics, vol.11, no.1, (2011), pp. 74-81.

[8] B. Singh, P. Jayaprakash, D. P. Kothari, et al., "Adaline-Based Control of Capacitor Supported DVR for Distribution System”, Journal of Power Electronics, vol.9, no. 8, (2009), pp. 386-395.

[9] V, Singh, S, C, Gupta, S, S, Pattnaik, et al., "Dynamic Voltage Restorer with New Hybrid Cascade Multilevel Inverter using Fuzzy Controller", International Journal of Computer Applications, vol. 111, no.7, (2015) , pp. 23-28.

[10] R. Omar, N.A. Rahim, A. Ahmad, et al., "Voltage Swells Improvement in Low Voltage Network Using Dynamic Voltage Restorer", American Journal of Applied Sciences, vol.8, no. 1, (2011), pp. 55-63.

[11] N. Liu, G. Yao, L. D. Zhou, et al., "Hळ loop shaping based robust controller of dynamic voltage restorer", Transactions of China Electrotechnical Society, vol. 27, no.9, (2012), pp. 48-53.

[12] J. F. Shi, "Research of Fuzzy-PID Temperature Control and Mathematical Modeling of Rice germination System Based on Genetic Algorithm", International Journal of Control and Automation, vol.9, no.4, (2016), pp. 51-62.

\section{Authors}

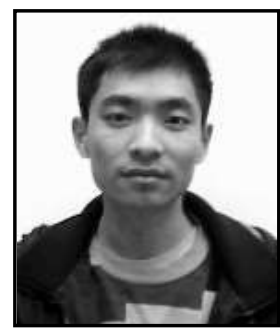

Penglong Yang, received his B. Sc. Degree in electric power and its automation from Henan University of Technology, China in 2014. He is now a master student at Ningbo University, China. His research interests include the analysis and control of power electronic systems, and the stability control of power systems.

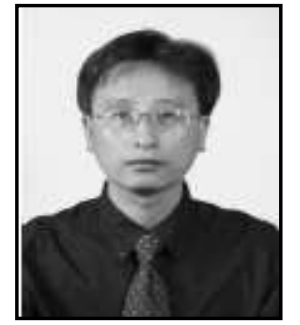

Wenlei Li, received his M.Sc. degree in electric power and its automation from Northeast DianLi University, Jilin, China in 1997, and $\mathrm{Ph} . \mathrm{D}$. degree in control theory and control engineering from Northeastern University, Shenyang, China in 2003.

$\mathrm{He}$ is now an associate professor with the Department of Electrical and Electronic Engineering at Ningbo University, Ningbo, China, and he was a visiting scholar with the Department of System and Computer Engineering, Carleton University, Ottawa, ON, Canada, from October, 2007 to September, 2008.

Dr. Li has published about 30 refereed journal and conference papers. His current research interests include nonlinear systems and 
control, stability control of power systems, MEMS system, as well as chaotic system and control.

E-mail: liwenlei@nbu.edu.cn (Corresponding author)

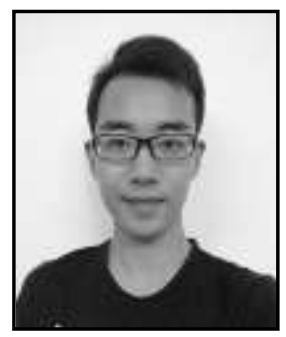

Jia Zhao, received his B. Sc. Degree in electrical engineering and its automation from Anhui Jianzhu University, China in 2015. He is now a master student at Ningbo University, China. His research interests include the analysis and control of power electronic systems.

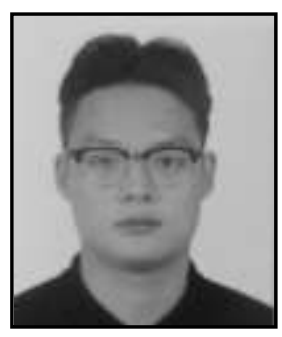

Yan Ding, received his bachelor's degree in electrical engineering and its automation from Ningbo University, Ningbo, China in 2016.

His current research interests include nonlinear systems and control, stability control of power systems.

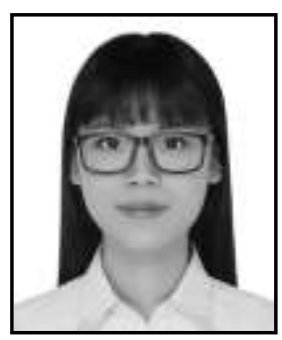

Jiayi Yuan, will received his bachelor's degree in electrical engineering and its automation from Ningbo University, Ningbo, China in 2017.

His current research interests include nonlinear systems and control, stability control of power systems. 
International Journal of Control and Automation Vol.10, No.7 (2017) 